\title{
SPECTRAL DETERMINATION OF ANALYTIC BI-AXISYMMETRIC PLANE DOMAINS
}

\author{
Steve ZeLDitCH
}

\begin{abstract}
Let $\mathcal{D}$ denote the class of bounded simply connected real analytic plane domains with the symmetry of an ellipse. We announce a proof that under generic assumptions, if $\Omega_{1}, \Omega_{2} \in \mathcal{D}$ and if the Dirichlet spectra coincide, $\operatorname{Spec}\left(\Omega_{1}\right)=\operatorname{Spec}\left(\Omega_{2}\right)$, then $\Omega_{1}=\Omega_{2}$ up to rigid motion.
\end{abstract}

\section{Introduction}

The purpose of this note is to announce and briefly describe a solution to the inverse spectral problem for the class $\mathcal{D}$ of domains $\Omega$ satisfying:

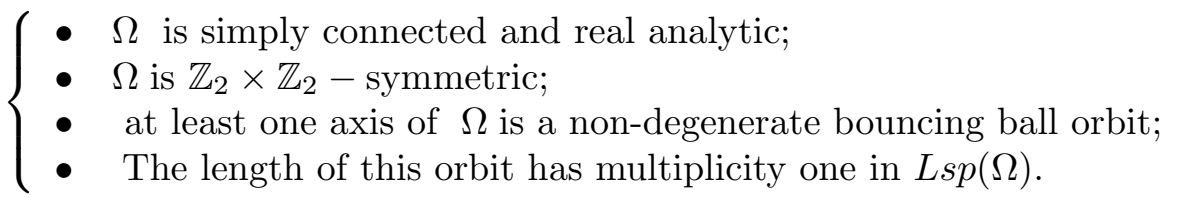

Denoting by $\operatorname{Spec}(\Omega)$ the spectrum of the Dirichlet Laplacian $\Delta_{\Omega}$ on $\Omega$, our result is:

Theorem 1.1. Spec: $\mathcal{D} \mapsto \mathbb{R}_{+}^{N}$ is 1-1.

Full details of the proof are contained in [Z.1]. In this note we will only describe the main ideas of the proof.

First let us clarify the assumptions on the domain. The symmetry assumption is that $\Omega$ is up/down symmetric across a horizontal axis and left/right symmetric across a vertical axis. Both axes intersect $\partial \Omega$ at right angles and hence define 'bouncing-ball' orbits of the billiard flow $G^{t}$ on $T^{*} \Omega$ with the usual reflection of trajectories at the boundary. That is, they are projections to $\Omega$ of periodic billiard trajectories with just two reflections at the boundary. We assume that at least one of these orbits $\gamma$ is non-degenerate in the sense that no eigenvalue of its (linear) Poincare map $P_{\gamma}$ is a root of unity. The orbit may be either elliptic or hyperbolic. With no loss of generality we can (and will) assume that $\gamma$ projects to the vertical axis $\overline{A B}$ and that the length of $\overline{A B}$ is $\pi$. Let us recall the definition of $P_{\gamma}$ : to any periodic billiard trajectory $\beta$ is associated its Poincare map $\mathcal{P}_{\beta}$, defined as the first return map on a local transversal to $\beta$ in $S^{*} \Omega$. A small neigborhood of $\overline{A B}$ in $\Omega$ is a strip with two boundary components, say $\Omega_{ \pm}$. The inward pointing unit tangent vectors to $\Omega$ along the lower boundary component

Received January 7, 1999.

Research partially supported by NSF grant \#DMS-9703775 
$\Omega_{-}$form a local transversal $S_{\partial \Omega_{-}}^{\text {in }}(\Omega)$, so we can regard $\mathcal{P}_{\gamma}$ as a symplectic map of $S_{\partial \Omega_{-}}^{i n}(\Omega)$ (with its usual symplectic structure). The derivative of $\mathcal{P}_{\gamma}$ at the unit tangent vector $\gamma(0)$ above the point $A$ is the linear Poincare map $P_{\gamma}$. We are assuming that $\gamma$ is either hyperbolic or non-degenerate elliptic; in the latter case, the eigenvalues of $P_{\gamma}$ have the form $\left\{e^{ \pm i \alpha}\right\}$ with $\alpha / \pi \notin \mathbb{Q}$. Finally, $\operatorname{Lsp}(\Omega)$ denotes the length spectrum of $\Omega$, i.e., the set of lengths of periodic billiard trajectories. The multiplicity assumption is that $\gamma$ is the unique periodic billiard trajectory of length $2 \pi$. For background on billiards and bouncing ball orbits in a related setting we refer to $[\mathrm{CV}][\mathrm{L}][\mathrm{B} . \mathrm{B}]$.

The proof of Theorem (1.1) is based on what may be called the 'strategy of Birkhoff normal forms.' By this we mean the approach to the inverse spectral theory of $\Delta$ on a Riemannian manifold $(M, g)$ (possibly with boundary) which is based on the following strategy (essentially due to V.Guillemin [G.1][G]):

- Introduce quantum normal forms for $\Delta$ at each closed geodesic $\gamma$;

- Prove that the normal forms are determined by $\operatorname{Spec}(\Delta)$;

- Determine the geometry of $(M, g)$ from the normal forms.

For a detailed exposition of normal forms and their applications to date in inverse spectral theory we refer to [Z.3] and [Z.5]. Let us only recall here that the strategy originated in Colin de Verdière's proof that domains in $\mathcal{D}$ (with $P_{\gamma}$ elliptic) are spectrally rigid $[\mathrm{CV}]$. He proved that domains in $\mathcal{D}$ with elliptic $P_{\gamma}$ are (i) determined by the Birkhoff normal form of the Poincare map $\mathcal{P}_{\gamma}$ at the bouncing ball orbit $\gamma$, and (ii) that the normal form of $\mathcal{P}_{\gamma}$ is rigid under deformations preserving $L s p(\Omega)$, and hence under isospectral deformations (satisfying generic conditions). Step (i) of Colin de Verdière's proof generalizes with no essential change of proof to the case of where $P_{\gamma}$ is hyperbolic. Hence it reduces the proof of Theorem (1.1) to the statement that the normal form of $\mathcal{P}_{\gamma}$ is a spectral invariant of $\Delta_{\Omega}$.

The latter statement is the main result of [Z.1]. As the table above suggests, the proof consists of two main steps. The first is to introduce a notion of quantum Birkhoff normal form of $\Delta$ around a bouncing ball orbit. The second is to prove that the normal form is a spectral invariant of $\Delta_{\Omega}$. It then follows easily that the classical Birkhoff normal form of $\mathcal{P}_{\gamma}$ is a spectral invariant. In the remainder of this announcement we will give a somewhat intuitive description of the normal form and a brief outline of the proofs.

A few final remarks to end the introduction. First, the restriction to Dirichlet boundary conditions is only for simplicity of exposition; the same result would hold for Neumann (among other) boundary conditions. Second, the assumption of bi-axisymmetry of the domain only plays a role in the third step of the strategy, i.e., in determining the domain from the normal form. The first two steps could be carried out for bouncing ball orbits (both elliptic and hyperbolic) of any domain. By analogy with analytic simple surfaces of revolution [Z.2] it seems reasonable to conjecture that analytic domains with just an up-down symmetry along a bouncing ball orbit should be determined by their spectra. 


\section{What is a quantum Birkhoff normal form around a bouncing ball orbit?}

Let us begin by recalling what is meant by the classical Birkhoff normal form of a Hamiltonian $H$ on a symplectic manifold $(X, \omega)$ at an equilibrium point $z$. To put $H$ in Birkhoff normal form around $z$ is to express $H \simeq F(I)$ as a function of locally defined 'action variables' $I$ centered at $z$. To put it actively, one wishes to construct a local symplectic transformation $\chi$ near $\gamma$ so that $\chi^{*} H \simeq F(I)$. In general, $H$ is not completely integrable near $z$, and the best one can do is to approximate $H$ by a completely integrable systems in small neighborhoods of $\gamma$ modulo errors which vanish to high order at $z$. That is, for each $N$ there exists $\chi_{N}$ on a neighborhood of $\gamma$ and a normal form $F_{N}$ so that $\chi_{N}^{*} H=F_{N}(I)+O\left(I^{N}\right)$ (where $z=\{I=0\}$.)

Our interest is actually in the case where $(X, \omega)$ is the cotangent bundle $T^{*} M$ of a surface $M$ and where $z$ is replaced by a closed geodesic $\gamma$ of a metric Hamiltonian. In this setting, the appropriate notion is of a homogeneous Birkhoff normal form, i.e., a normal form which is homogeneous with respect to the $\mathbb{R}^{+}$ action on $T^{*} M-0$ given by $r(x, \xi)=(x, r \xi)$. This normal form is described in detail in [F.G] for elliptic closed orbits in the boundaryless case. A neighborhood of an embedded ( orientable) closed geodesic is a tube diffeomorphic to $S^{1} \times \mathbb{R}$. Hence one can use this as the model configuration space and $T^{*}\left(S^{1} \times \mathbb{R}\right)$ as the model phase space. There is a distinguished homogeneous action variable $\sigma$, namely the symplectic coordinate dual to the arclength coordinate on $\gamma$. In the transverse direction to $\gamma$ one introduces a homogeneous action variable $I_{j}$. In the elliptic case it is the 'harmonic oscillator' $I^{e}=\frac{1}{2}\left(\sigma y^{2}+\sigma^{-1} \eta^{2}\right)$ while in the hyperbolic case it is the hyperbolic action $I^{h}=\frac{1}{2}\left(-\sigma y^{2}+\sigma^{-1} \eta^{2}\right)$. Then there exists a homogeneous symplectic transformation $\chi$ defined in a conic neighborhood $V$ of $\gamma$ so that $\chi^{*} H$ is approximately given by the homogeneous (degree one) Birkhoff normal form

$$
F=\sigma+\frac{\alpha}{L} I+\frac{p_{1}(I)}{\sigma}+\frac{p_{2}(I)}{\sigma^{2}}+\ldots
$$

where $p_{j}$ is a homogeneous polynomial of degree $j+1$. We refer to [F.G][G.1] [G] [Z.3] for a detailed discussion.

Now let us move on to domains with boundary. A natural model for a neighborhood of a bouncing ball orbit is the strip $\Omega_{o}:=[0, L] \times \mathbb{R}$. The bouncing ball orbit will be the broken geodesic $\gamma_{o}$ projecting to the segment $[0, L] \times\{0\}$. One can use precisely the same definition of action variables as in the boundaryless case. In coordinates $(s, y) \in[0, L] \times \mathbb{R}$ on the model domain and dual coordinates $(\sigma, \eta)$ on the fibers of $\left.T^{*}\left(\mathbb{R}^{2}\right)\right|_{[0, L] \times \mathbb{R}}$ we may define the action variables as $\{\sigma, I\}$ and normal forms $F(\sigma, I)$ just as in the boundaryless case. They generate broken Hamiltonian flows on $T^{*} \Omega_{o}$ with the usual law of reflection at the boundary. The action coordinates and normal form are actually only defined in a conic neighborhood $V$ of the bouncing ball orbit $\gamma_{o}$ given by $V=V^{+} \cup V^{-}$ 
where $V^{ \pm}=\{(s, \pm \sigma, y, \eta):|y| \leq \epsilon,|\eta| \leq \epsilon \sigma, \sigma>0\}$.

We now proceed to quantum Birkhoff normal forms. As in [Z.4], our approach to the quantum Birkhoff normal form of $\Delta_{\Omega}$ at a bouncing ball orbit operates entirely at the quantum level, i.e., we do not first construct a classical normal form for the symbol of $\Delta$ and then quantize it (as is done in [G] for instance). By definition, a quantum Birkhoff normal form is a function of certain commuting operators $(|D|, \hat{I})$ on the model space, which one thinks of as quantized actions. The first one is $\left|D_{s}\right|=|D|$, the square root of the Dirichlet Laplacian on $[0, \pi]$, extended in the obvious way as an operator on $\Omega_{o}$, i.e., $|D|$ is the operator defined by $|D| \sin (k s) f(y)=|k| \sin (k s) f(y)$. The second is the quantum action operator on the transverse space. In the elliptic case, it is the harmonic oscillator $\hat{I}^{e}=\frac{1}{2}\left(|D|^{-1} D_{y}^{2}+|D| y^{2}\right)$ while in the hyperbolic case it is given by $\hat{I}^{h}=$ $\frac{1}{2}\left(|D|^{-1} D_{y}^{2}-|D| y^{2}\right)$. It is well-defined microlocally in $V$ since $\sigma \neq 0$ in $V$ and $|D|$ is microlocally invertible. The operators $|D|, \hat{I}$ commute and hence have a joint spectrum of eigenvalues/eigenfunctions. In the elliptic case, the joint spectrum is discrete, with eigenfunctions given by $\phi_{k q}(s, y):=\sin (k s) D_{q}(\sqrt{k} y)$ where $D_{q}(y)$ is the normalized Hermite function $C_{q} H_{q}(y) e^{-\frac{1}{2} y^{2}}$, with $H_{q}$ the qth Hermite polynomial and $C_{q}$ a normalizing constant. In the hyperbolic case the joint spectrum is continuous. The hyperbolic action is unitarily equivalent to $\frac{1}{2}\left(y D_{y}+D_{y} y\right)$, which has generalized eigenfunctions $Y_{r}(y)=y^{-\frac{1}{2}+i r}$ so the joint eigenfunctions of $|D|, \hat{I}$ may be taken in the form $\sin k s Y_{r}(y)$.

By a first order quantum normal form we mean a first order polyhomogeneous symbol $F(|D|, \hat{I})$ in $|D|, \hat{I}$ with an asymptotic expansion of the form:

$$
F(|D|, \hat{I}) \sim|D|+\frac{\alpha}{\pi} \hat{I}+\frac{p_{1}(\hat{I})}{|D|}+\frac{p_{2}(\hat{I})}{|D|^{2}}+\ldots,
$$

where $p_{j}$ a polynomial of degree $j+1$. The coefficients $B_{\gamma k}$ of the polynomials $p_{j}$ are known as the normal form coefficients. The remainder is assumed to consist of terms which in some combination either have a very low pseudodifferential order or which vanish to a high order along $\gamma_{o}$. Technically, $F$ belongs to the (Boutet de Monvel) class $S^{m, k}\left(V, \mathbb{R}^{+} \gamma_{o}\right)$ of symbols admitting an asymptotic expansion of the form $a(\sigma, I) \sim \sum_{j=0}^{\infty} a_{m-j}(\sigma, I), a_{m-j}(r \sigma, r I)=r^{m-j} a(\sigma, I)$ with $a_{m-j} \in O_{k-2 j} S^{m-j}$. Here, $O_{k-2 j} S^{m-j}$ is the class of symbols of order $m-j$ which vanish to order $2 k-j$ at $I=0$. Thus, we assume $F \in S^{1,0}$ and that the remainder after $K$ terms lies in $S^{1, K}$.

\section{How to construct the quantum normal form}

Speaking heuristically, we would like to conjugate $\sqrt{\Delta}$ near $\overline{A B}$ to a quantum Birkhoff normal form $F(|D|, \hat{I})$ at $\gamma_{o}$. Since $\Delta$ lives on $\Omega$ the first issue is how to transport it to the model domain $\Omega_{o}$.

In fact, a special map $\Phi: \Omega \rightarrow \Omega_{o}$ was introduced by Lazutkin in [L] for a closely related purpose. Lazutkin's goal was to construct a large family of quasimodes $u_{k q}$ associated to an elliptic bouncing ball orbit of a plane domain. 
Such quasimodes are approximate eigenfunctions of the Laplacian, i.e., they satisfy

$$
\Delta u_{k q} \approx \lambda_{k q}^{2} u_{k q}, \quad \lambda_{k q} \approx \frac{1}{L}\left(\pi k+\alpha\left(q+\frac{1}{2}\right)\right)+\frac{p_{1}(q)}{k}+\ldots
$$

Here, $2 L$ is the length of the orbit (recall that we set $L=\pi$ ). We observe that the form of $\lambda_{k q}$ is precisely what we defined above (in operator form) as a quantum normal form. Hence the construction of quasimodes is of some value as a guide to the construction of a normal form. It should be noted that the intertwining operator to normal exists in the hyperbolic case even though one cannot associated quasimodes to hyperbolic orbits.

Taking the lead from [L], we first use a Lazutkin-type map $\Phi$ to straighten the domain near $\overline{A B}$ to $\Omega_{o}$ while carrying $\Delta$ to a variable coefficient Laplacian $\bar{\Delta}$. Such a map exists also in the hyperbolic case. In the elliptic cases, the new Laplacian has the form $\bar{\Delta} \sim D_{\bar{s}}^{2}+B(\bar{s}, \bar{y})\left(\bar{y}^{2} D_{\bar{s}}^{2}+D_{\bar{y}}^{2}\right)+\Gamma_{s}^{\prime} D_{s}+\Gamma_{y}^{\prime} D_{y}$, while in the hyperbolic case the second term has the form $B(\bar{s}, \bar{y})\left(-\bar{y}^{2} D_{\bar{s}}^{2}+D_{\bar{y}}^{2}\right)$. Here, $B(s, y)$ is a power series in $y$ with analytic coefficients in $s \in(-\epsilon, L+$ $\epsilon)$. We then wish to conjugate it by a Fourier integral operator into normal form. Almost immediately, however, the complication of the boundary is felt. Pseudodifferential and Fourier integral operators on manifolds with boundary are very complicated objects and it is not clear that the classes so far introduced are even relevant to our problem. Hence we would like to avoid dealing with them. To do so, we will work in the open space $S^{1} \times \mathbb{R}$ containing $\Omega_{o}$ and only use the boundary to put boundary conditions on our operators. Here, $S^{1}=\mathbb{R} / 2 \pi \mathbb{Z}$.

What we seek then is a microlocally unitary FIO (Fourier integral operator) $W$ on the open space which conjugates $\bar{\Delta}$ to a second order normal form, i.e., the square of $F$ above, modulo acceptable errors. Additionally, $W$ should preserve Dirichlet boundary conditions along $\partial \Omega_{o}$ in the sense that $W \phi_{k q}$ should vanish on $\partial \Omega_{o}$. The following lemma completes the first step of the normal forms strategy. We use the notation $A \sim B$ in $V$ to mean that $A-B$ is a Fourier integral operator of order $-\infty$ in $V$.

Lemma 3.1. Assume that $\gamma_{o}$ is a non-degenerate bouncing ball orbit. Then for each $K>0$, there exists a microlocally invertible Fourier integral operator $W$ with (two-sided) microlocal inverse $W_{-1}$ defined in a conic neighborhood of $\gamma_{o}$ in $T^{*}\left(S_{2 L}^{1} \times \mathbb{R}\right)$ such that:

(i) $W_{-1} \bar{\Delta} W \sim\left[F(|D|, \hat{I})^{2}+E^{K}\right]$ in $V$.

(ii) $W F(|D|, \hat{I})^{2} W_{-1}=\left[\bar{\Delta}-W E^{K} W_{-1}\right]$ in $V$.

(iii) If $u \in H_{0}^{1}\left(\Omega_{o}\right)$ then $W u=0$ on $\partial \Omega_{o}$.

(iv) The symbol of the remainder term $E^{K}$ belongs to $S^{2, K}\left(V, \mathbb{R}^{+} \gamma_{o}\right)$.

A few words about the remainder term and the proof. As mentioned above, (iv) means that the symbol of $E^{K}$ consists of terms which either vanish to high order along $\gamma$ or have a very negative pseudodifferential order in $V$ (or some 
combination of both). In the next section we will see that such terms form an acceptable remainder. Regarding the proof of lemma (3.1), it follows the approach given in [Z.4] in the boundaryless case. $W$ is constructed by gluing together component operators $W_{k}$ which only act on Fourier components $\sin (k s) f(y)$. The $W_{k}$ can be constructed as products of exponentials $e^{k^{-j / 2}(P+i Q)_{j / 2}}$ of semiclassical pseudodifferential operators $(P+i Q)_{j / 2}$ on the transverse space $\mathbb{R}$, where the Weyl symbols of $P_{j / 2}$ and $Q_{j / 2}$ are assumed to be real valued. It turns out that the top order homogeneous part of $P_{j / 2}$ vanishes, so $P$ only adds a zeroth order pseudodifferntial term to the exponent of the 'glued' Fourier integral operator. As in [Z.4] (or in other constructions of Birkhoff normal forms), the condition that $W_{k}$ be an intertwining operator to normal form translates into a series of homological (or transport) equations for the symbols of $P_{j / 2}, Q_{j / 2}$. These are first order equations, and the initially mysterious aspect is that one can solve the equations with boundary conditions at two boundary components $(s=0, \pi)$. The resolution of this mystery is that the boundary conditions only apply to the 'odd' part of the symbol of $P_{j / 2}$ and to the 'even' part of the symbol of $Q_{j / 2}$ under time reversal. The remaining parts automatically satisfy the boundary conditions. After solving for the even part of $P_{j / 2}$ in terms of the odd part $P_{j / 2}^{o}$, resp. the odd part of $Q_{j / 2}$ in terms of the even part $Q_{j / 2}^{e}$, one gets sec-

ond order Sturm-Liouville equations for the symbols $P_{j / 2}^{o}, Q_{j / 2}^{e}$ and when $\gamma_{o}$ is non-degenerate, these equations can be solved.

\section{Spectral invariance of the normal form}

We now explain how to carry out the second step of proving that the normal form at $\overline{A B}$ is a spectral invariant of $\Delta_{\Omega}$.

The link between the spectrum and normal form is through the coefficients in the singularity expansion of the wave trace [GM]. In the case of a bouncing ball orbit (or more generally of a transversally periodic reflecting ray) $\gamma$ whose length $L_{\gamma}$ is isolated and of multiplicity one in $L s p(\Omega)$, the trace of the Dirichlet wave group $E(t)=\cos \left(t \sqrt{\Delta}_{\Omega}\right)$ near $t=m L_{\gamma}$ has the singularity expansion

$$
\begin{aligned}
\operatorname{Tr} \psi E(t)=c_{\gamma^{m}}\left(t-m L_{\gamma}+i 0\right)^{-1}+a_{\gamma^{m} 0} \log \left(t-m L_{\gamma}+i 0\right)+ \\
\\
\sum_{k=1}^{\infty} a_{\gamma^{m} k}\left(t-m L_{\gamma}+i 0\right)^{k} \log \left(t-m L_{\gamma}+i 0\right),
\end{aligned}
$$

where the coefficients $a_{\gamma^{m} k}$ are calculated by the stationary phase method from a Lagrangean parametrix. Here, $\gamma^{m}$ denotes the mth iterate of $\gamma$ and $\psi$ is a cutoff to a microlocal neighborhood of $\gamma$.

As in the boundaryless case, we can identify the wave invariants as noncommutative residues (cf., [G][Z.1]). Recall that if $A$ is a Fourier integral operator, and if $P$ is any positive elliptic first order pseuodifferential operator, then the zeta function $\zeta(z, A, P):=\operatorname{Tr} A P^{-z}$ has meromorphic extension to $\mathbb{C}$ with at most simple poles. The residue at $z=0$ is referred to as the 
non-commutative residue $\operatorname{res}(A)$ of $A$. It is independent of $A$ and is a tracial invariant, i.e., res $\left(W A W^{-1}\right)=\operatorname{res} A$. In the boundaryless case, one has $a_{\gamma k}=\left.\operatorname{res}\left(\frac{d}{d t}\right)^{k} E(t)\right|_{t=L_{\gamma}}$. The only ingredients in the proof are the Lagrangean property of $E$ and a canonical transform between $\operatorname{Tr} A e^{i t P}$ and $\operatorname{Tr} A P^{-s}$. Hence the same result remains valid in the case of periodic reflecting rays of the boundary case:

Corollary 4.1. If $\gamma$ is a periodic reflecting ray, then $a_{\gamma k}=\left.\operatorname{res}\left(\frac{d}{d t}\right)^{k} E(t)\right|_{t=L_{\gamma}}$.

The main lemma in the proof of theorem (1.1) can now be stated. Let $\gamma_{o}$ be the bouncing ball orbit of the normal form on the model domain, i.e., $\gamma_{o}$ projects to $[0, \pi] \times\{0\}$.

Lemma 4.2. Let $\gamma$ be a non-degenerate bouncing ball orbit. Then for all $m$, we have: $a_{\gamma^{m} k}\left(\sqrt{\Delta_{\Omega}}\right)=a_{\gamma_{o}^{m} k}(F(|D|, \hat{I}))$. Hence, the quantum Birkhoff normal coefficients of $\Delta_{\Omega}$ at $\gamma$ are spectral invariants of $\Delta_{\Omega}$.

The basic idea of the proof of lemma (4.2) is to use the intertwining operator $W$ and the normal form $F$ of $\sqrt{\bar{\Delta}}$ to construct a parametrix $W F_{o}(t) \psi_{V} 1_{\Omega_{o}} W_{-1}$ for the Dirichlet wave group $E(t)$. Here, $F_{o}(t)$ is the 'odd part' of the wave group generated by the normal form, which by construction satisfies Dirichlet boundary conditions at $s=0, L$. Also, $\psi_{V}$ is a microlocal cutoff to $V$ and $1_{\Omega_{o}}$ is the characteristic function of $\Omega_{0}$. The parametrix only approximates $E(t)$ modulo errors with symbols in $S^{2,2(k+2)}\left(V, \mathbb{R}^{+} \gamma\right)$. However, such errors are irrelevant because of the following:

Proposition 4.3. $a_{\gamma k}(\bar{\Delta})$ depends only on the class of $\bar{\Delta}$ modulo $O p^{w} S^{2,2(k+2)}\left(V, \mathbb{R}^{+} \gamma\right)$.

It follows that the parametrix has the same wave trace invariants as the Dirichlet wave group $E(t)$. Then, using the tracial properties of the residue, one can further show that the model Dirichlet wave group $F_{o}(t)$ has the same wave trace invariants as $E(t)$. To complete the proof of lemma (4.2) it suffices to show that the normal form $F$ is determined by the wave invariants of $F_{o}(t)$. This goes precisely as in the boundaryless case [G] [Z.4]. The proof comes down to to determining the coefficients of a constant coefficient differential operator from its action on exponential polynomials.

To complete the proof of theorem (1.1), it remains to observe that a Birkhoff normal form for $H$ at $\gamma$ induces a Birkhoff normal form of the Poincare map $\mathcal{P}_{\gamma}$. Hence the latter is a spectral invariant. By the result of Colin de Verdière a domain in $\mathcal{D}$ is determined by this normal form in the non-degenerate case. The proof extends with no serious incidents to the hyperbolic case and hence the domain is determined in the general non-degenerate case. 


\section{References}

[B.B] V.M. Babic and V.S. Buldyrev, Short-wavelength diffraction theory, Springer Series on Wave Phenomena, 4, Springer-Verlag, New York (1991).

[CV] Y. Colin de Verdiere, Sur les longuers des trajectoires périodiques d'un billard, In: P. Dazord and N. Desolneux-Moulis (eds.) Geometrie symplectique et de contact: autour du theoreme de Poincare-Birkhoff, Sem. Sud-Rhodanien de Geometrie III, Travaux en Cours, 122-139, Hermann, Paris, 1984. 122-139.

[F.G] J.P. Françoise and V. Guillemin, On the period spectrum of a symplectic mapping, J. Funct. Anal. 100 (1991), 317-358.

[G] V. Guillemin, Wave trace invariants, Duke Math J. 83 (1996), 287-352.

[G.1] , Wave-trace invariants and a theorem of Zelditch, Internat. Math. Res. Notices 12 (1993), 303-308.

[GM] V. Guillemin and R.B. Melrose, The Poisson summation formula for manifolds with boundary, Adv. in Math. 32 (1979), 204-232.

[HoIV] L. Hörmander, The analysis of linear partial differential operators IV, Fourier integral operators, Grundlehren der mathematischen Wissenschaften, 275, Springer-Verlag, Berlin-New York, 1985.

[L] V.F. Lazutkin, Construction of an asymptotic series of eigenfunctions of the "bouncing ball" type, Asymptotic methods and stochastic models in wave propagation problems, Trudy Mat. Inst. Steklov. 95 (1968), 125-140.

[Z.1] S. Zelditch, The inverse spectral problem for biaxi-symmetric analytic plane domains, preprint, 1998.

[Z.2] _ The inverse spectral problem for surfaces of revolution, J. Diff. Geom. 49 (1998), 207-264.

[Z.3] Normal form of the wave group and inverse spectral theory, Journées Équations aux Dérivées Partielles, Saint-Jean-de-Monts, 1998.

[Z.4] Wave invariants at elliptic closed geodesics, Geom. Funct. Anal. 7 (1997), 145-213.

[Z.5] Lectures on wave invariants, Proc. ICMS, (Y. Safarov, ed.), to appear.

Department of Mathematics, Johns Hopkins University, Baltimore, MD 21218 , USA

E-mail address: zel@math.jhu.edu 